Cahiers de la recherche sur les droits

Cahiers

${ }^{\text {sur les }}$ Droits fondamentaux

$9 \mid 2011$

Conseil constitutionnel et droits fondamentaux

\title{
Avant la naissance et après la mort : l'être humain, une chose digne de respect
}

Amandine Cayol

\section{(2) OpenEdition}

\section{Journals}

Édition électronique

URL : https://journals.openedition.org/crdf/5450

DOI : $10.4000 /$ crdf.5450

ISSN : 2264-1246

Éditeur

Presses universitaires de Caen

Édition imprimée

Date de publication : 1 décembre 2011

Pagination : 117-126

ISBN : 978-2-84133-396-7

ISSN : 1634-8842

\section{Référence électronique}

Amandine Cayol, « Avant la naissance et après la mort : l'être humain, une chose digne de respect », Cahiers de la recherche sur les droits fondamentaux [En ligne], 9 | 2011, mis en ligne le 01 décembre 2012, consulté le 15 février 2023. URL : http://journals.openedition.org/crdf/5450 ; DOI : https:// doi.org/10.4000/crdf.5450 


\title{
Avant la naissance et après la mort: I'être humain, une chose digne de respect ${ }^{1}$
}

\author{
Amandine CAYOL \\ Maître de conférences en droit privé à l'université de Caen Basse-Normandie
}

I. Le respect dû à l'être humain avant la naissance

A. L'absence de personnalité juridique du fœtus

B. Le fœtus, un être humain digne de respect

II. Le respect dû à l'être humain après la mort
A. Le cadavre, une chose sacrée
B. Le défunt, un être humain digne de respect

Zénon enseigne à l'homme qu'il a une dignité, non de citoyen, mais d'homme; qu'outre ses devoirs envers la loi, il en a envers lui-même ${ }^{2}$.

Longtemps absent des textes de droit interne, le principe de dignité de la personne humaine a été consacré dans la loi française en 1994. La loi relative au respect du corps humain du 29 juillet 1994 a inséré un article 16 dans le Code civil, aux termes duquel «la loi interdit toute atteinte à la dignité humaine». Le nouveau Code pénal, entré en vigueur la même année, contient, quant à lui, un chapitre intitulé «Des atteintes à la dignité humaine». Le respect de la dignité humaine a immédiatement été érigé par le Conseil constitutionnel en principe à valeur constitutionnelle dans sa décision "Bioéthique» du 27 juillet 1994. Il y affirme clairement que «la sauvegarde de la dignité de la personne humaine contre toute forme d'asservissement et de dégradation est un principe à valeur constitutionnelle» ${ }^{3}$.

S'il a tardé à être expressément reconnu en droit français, le principe de dignité humaine a été affirmé, au plan international, dès la fin de la seconde guerre mondiale 4 . Après les atrocités du régime nazi, il est apparu nécessaire de rappeler que la dignité est inhérente à tous les membres de la famille humaine. Tout ce qui tend à déshumaniser l'homme est une atteinte à sa dignité. Les crimes commis pendant la seconde guerre mondiale, consistant à nier la qualité d'être humain de certaines catégories de

1. Cet article a été écrit avant la réforme issue de la loi du 7 juillet 2011. Celle-ci ne procède qu'à des aménagements des règles préexistantes sur les points abordés dans cet article.

2. Numa Denis Fustel de Coulanges, La Cité antique [1864], Paris, Librairie Hachette, 1927, p. 423.

3. Déc. $n^{\circ}$ 94-343/344 DC du 27 juillet 1994, JO du 29 juillet 1994, p. 11024.

4. Pour un historique de la notion, voir M.-L. Pavia, «La découverte de la dignité humaine», in La Dignité de la personne humaine, M.-L. Pavia et T. Revet (dir.), Paris, Economica, 1999, p. 3-23. 
personnes, apparaissaient hors norme, hors du commun. Ils appelaient la création d'une infraction particulière et imprescriptible: le crime contre l'humanité. Parallèlement, de nombreux textes ont réaffirmé la dignité de tout être humain. Dans le préambule de la Charte des Nations unies, signée le 26 juin 1945, les peuples des Nations unies proclament leur foi «dans les droits fondamentaux de l'homme, dans la dignité et la valeur de la personne humaine [...] ». Le préambule de la Déclaration universelle des Droits de l'homme des Nations unies du 10 décembre 1948 consacre également la «reconnaissance de la dignité inhérente à tous les membres de la famille humaine». La dignité n'est pas attribuée mais intrinsèque aux personnes. Les textes qui la proclament sont déclaratifs et non constitutifs. Tel est le cas de la Constitution de plusieurs pays d'Europe occidentale $^{5}$. La Loi fondamentale pour la République fédérale d'Allemagne affirme ainsi avec force le concept de dignité. On peut lire, dès le premier alinéa de son article premier, que «la dignité de l'être humain est intangible. Tous les pouvoirs publics ont l'obligation de la respecter et de la protéger». La Constitution française ne consacre cependant pas un tel principe, malgré plusieurs propositions de modification en ce sens ${ }^{6}$.

Affirmée en réaction à la barbarie nazie, la notion de dignité humaine a été de nouveau mobilisée face aux avancées de la médecine ${ }^{7}$. L'homme peut désormais, à l'image de Dieu, produire d'autres êtres humains. Ces hommes "produits» ne risquent-ils pas d'être traités comme de simples choses? Dans le cadre de la procréation médicalement assistée, les embryons fabriqués et congelés en attente d'un éventuel projet parental sont-ils encore des êtres humains? Leur destruction automatique au bout de cinq ans n'exclut-elle pas une telle qualification? La recherche sur les embryons ne les transforme-t-elle pas en simples objets? La naissance, le 26 janvier 2011, du premier «bébé-médicament» français relance le débat. Il peut certes paraître miraculeux de pouvoir ainsi donner naissance par fécondation in vitro à un enfant permettant de soigner un de ses aînés pour lequel il est un donneur compatible. Toutefois la production de tels enfants, après double diagnostic préimplantatoire permettant le choix des embryons, ne les réduit-elle pas au rang de simples moyens, de choses ayant pour seule vocation de servir les intérêts d'autrui?

Le principe de dignité humaine a été maintes fois rappelé face aux risques de réification de la personne que présentent les progrès scientifiques. Dans la lignée de la philosophie kantienne, l'homme est vu comme une fin en soi et ne saurait en aucune façon être rabaissé au rang de moyen. On ne doit pas traiter l'homme comme un objet, l'instrumentaliser, c'est-à-dire l'utiliser à une fin qui lui est étrangère ${ }^{8}$. Les textes de «bioéthique» se multiplient. L'article premier de la Convention du Conseil de l'Europe pour la protection des Droits de l'homme et de la dignité de l'être humain à l'égard de l'application de la biologie et de la médecine, adoptée à Oviedo le 4 avril $1997^{9}$, indique ainsi que «les parties à la présente Convention protègent l'être humain dans sa dignité et son identité et garantissent à toute personne, sans discrimination, le respect de son intégrité et de ses autres droits et libertés fondamentales à l'égard des applications de la biologie et de la médecine».

Le protocole additionnel du 12 janvier 1998 précise que «l'instrumentalisation de l'être humain par la création délibérée d'êtres humains génétiquement identiques est contraire à la dignité de l'homme et constitue un usage impropre de la biologie et de la médecine». L'interdiction du clonage humain est donc posée par son article premier. Le Code de la santé publique impose aux médecins d'exercer leur «mission dans le respect de la vie humaine, de la personne et de sa dignité ${ }^{10}$.

Le principe de dignité de la personne humaine est conçu comme le rempart contre tout risque de réification de la personne. Tout être humain, du fait de sa dignité, doit être respecté. Principe à valeur constitutionnelle, la dignité est aussi un principe fondamental au niveau européen. La Convention européenne de sauvegarde des Droits de l'homme et des libertés fondamentales ne consacre pas explicitement le principe de dignité. Toutefois, la Cour européenne des Droits de l'homme considère qu'il constitue l'essence même des objectifs fondamentaux protégés par la Convention ${ }^{11}$. La Charte des droits fondamentaux de l'Union européenne, proclamée lors du sommet de Nice du 7 décembre $2000^{12}$, y consacre quant à elle son

5. Article 1 de la Constitution portugaise, article 10 de la Constitution espagnole, article 23 de la Constitution belge, article 4 de la Constitution bulgare, article 1 de la Constitution de la République tchèque, article 19 de la Constitution slovaque, article 21 de la Constitution lituanienne, article 1 de la Constitution lettone, article 21 de la Constitution russe, article 30 de la Constitution polonaise.

6. Le rapport du Comité présidé par Simone Veil, remis au président de la République le 8 décembre 2008, proposait de consacrer le principe de dignité dans la Constitution. Il n’a pas été suivi d'effets. Une telle suggestion avait déjà été faite en 1993 par le Comité consultatif pour la révision de la Constitution, présidé par le doyen Georges Vedel (JO du 16 février 1993).

7. M. Zabaloueff, «Bioéthique et dignité humaine», in La Dignité, J.-M. Pontier (dir.), Aix-en-Provence, Presses universitaires d'Aix-Marseille, 2003, p. 35 sq.

8. B. Mathieu, «Les droits fondamentaux: les contraintes (?) du droit international et du droit constitutionnel ", Revue générale de droit médical, $\mathrm{n}^{\circ}$ spécial, La recherche sur l'embryon: qualification et enjeux, 2000, p. 217.

9. Sur ce texte, voir notamment P. Fraisseix, «La protection de la dignité de la personne et de l'espèce humaines dans le domaine de la biomédecine: l'exemple de la Convention d'Oviedo", in L'évolution des droits fondamentaux de la personne humaine en 1997 et 1998, G. Lebreton (dir.), Paris, L'Harmattan, 200o, p. 119-130.

10. Article R. 4127-2 du Code de la santé publique.

11. CEDH, 22 novembre 1995, SW c. RU.

12. Sur cette charte, voir A. Renaut-Couteau, "Analyse de la Charte des droits fondamentaux de l'UE", in Regards critiques sur l'évolution des droits fondamentaux de la personne humaine en 1999 et 2000, G. Lebreton (dir.), Paris, L'Harmattan, 2002, p. 75-102. 
article premier, aux termes duquel «la dignité humaine est inviolable. Elle doit être respectée et protégée».

Encore faut-il déterminer quels sont les individus dignes de respect. Que désigne le concept de «personne humaine» souvent utilisé dans les textes relatifs à la dignité? Qui sont les «membres de la famille humaine» ? S'il ne fait guère de doute que toutes les personnes physiques vivantes sont visées, doit-on également y inclure les enfants simplement conçus? Que penser du statut des personnes décédées? La mort les ramène-t-elle au simple rang de chose? Une analyse plus poussée des notions juridiques de chose et de personne s'impose.

Depuis le droit romain, il est d'usage d'opposer les personnes et les choses: tout ce qui n'est pas une personne est une chose. Traditionnelle, cette summa divisio semble pourtant de plus en plus souvent remise en cause aujourd'hui. Entre réification de la personne humaine et personnification de certaines catégories de choses, ses frontières semblent ténues... Le contrat de travail ne conduit-il pas à une certaine réification de la personne? Le salarié s'engageant à mettre sa force de travail à la disposition de son employeur ne loue-t-il pas une partie de sa personne? La subordination juridique est en effet le moyen d'objectiver la force de travail du salarié, de la réifier afin de lui permettre d'être l'objet d'un contrat. Si le corps humain en son entier ne peut se distinguer de la personne ${ }^{13}$, des éléments détachés de celui-ci deviennent des choses ${ }^{14}$. Les cheveux, le sang ou même les organes sont ainsi, juridiquement, des choses. Parallèlement, des choses rattachées au corps humain peuvent être qualifiées de personnes par destination. Tel a pu être le cas de prothèses ${ }^{15}$, ou même de chiens d'aveugles ${ }^{16} \ldots$

L'accélération des innovations scientifiques et les progrès de la médecine bouleversent la notion de personne. L'enfant simplement conçu est-il déjà une personne? Peuton librement produire des embryons, les céder, procéder sur eux à des expérimentations, voire les détruire? Estil concevable de produire un homme pour en soigner un autre? Pendant longtemps, l'impossible faisait figure d'interdit, mais le champ des possibles s'étendant, il devient nécessaire de poser des limites et, pour cela, de définir précisément les notions. Telle est la fonction du droit. Qu'est-ce qu'une personne? La question est aujourd'hui soulevée avec la plus grande acuité.

Il convient de distinguer clairement les notions de personne juridique et de personne humain $\mathrm{e}^{17}$. Deux ordres de la personnalité coexistent: celui de la réalité, dont relève la personne humaine concrète, et celui du concept juridique, dont relève la personnalité juridique ${ }^{18}$. Cette dernière confère la qualité de sujet de droit, d'acteur sur la scène juridique: elle permet d'avoir un patrimoine, de conclure des contrats, d'ester en justice... À l'origine, le terme persona désigne ainsi le masque qui permet à l'acteur, au théâtre, de jouer le rôle désigné par le masque. Abstraite, la personnalité juridique n'est pas réservée aux seuls êtres humains ${ }^{19}$. Elle peut notamment être accordée à des groupements, auxquels le droit positif reconnaît la qualité de personnes morales sous certaines conditions. «La personnalité juridique n'est pas une qualité que l'homme posséderait par nature mais que lui attribue le droit ${ }^{20}$. Au contraire, la qualité d'être humain est un fait, une réalité qui ne requiert aucune décision juridique en ce sens. Elle n'est pas octroyée par le droit, mais seulement constatée.

La dignité humaine renvoie à ce qu'il y a d'humain dans l'homme, à l'essence de la personne humaine. Visant à protéger la personne humaine, et non la personne juridique, la notion de dignité est applicable à des êtres humains n'ayant pas encore, ou n'ayant plus, la qualité de personne juridique $^{21}$. Le fotus, tout comme le cadavre, sont, en droit positif, des choses. Avant la naissance (I) et après la mort (II), l'être humain est une chose digne de respect ${ }^{22}$.

\section{Le respect dû à l'être humain avant la naissance}

Avant la naissance, aucune personne juridique n'est reconnue par le droit français (A). Il n'en demeure pas moins qu'un être humain existe dès la conception, et qu'un certain respect lui est dû (B).

13. I. Moine, Les choses hors commerce. Une approche de la personne humaine juridique, Paris, LGDJ (Bibliothèque de droit privé, t. 271), $1997, \mathrm{p} .119$. Contra J.-P. Baud, L'affaire de la main volée. Une histoire juridique du corps, Paris, Seuil, 1993, p. 44

14. E. Sabathié, La Chose en droit civil, Thèse, Paris II, 2004, $\mathrm{n}^{\circ}$ 659, p. 608. R. Libchaber, «Biens», Répertoire civil Dalloz, septembre 2002 , p. 15 , $\$ 79$.

15. Cass. $1^{\text {re }}$ civ., 11 décembre 1985, Bull. civ., $\mathrm{n}^{\circ} 348$ : la prothèse fait "partie intégrante de la personne humaine ".

16. X. Labbée, «Le chien prothèse», Dalloz, $\mathrm{n}^{\circ} 36,2000$, p. 750.

17. Certains auteurs semblent confondre les deux. Voir, par exemple, M. Herzog-Evans, «Homme, homme juridique et humanité de l'embryon», RTD civ., 2000, p. 65-78.

18. A. Bertrand-Mirkovic, La notion juridique de personne (étude visant à clarifier le statut juridique de l'enfant à naître), Aix-en-Provence, Presses universitaires d'Aix-Marseille, 2003, p. 259, $\mathrm{n}^{\circ}$ 508. R. Andorno, La distinction juridique entre les personnes et les choses. À l'épreuve des procréations artificielles, Paris, LGDJ (Bibliothèque de droit privé, t. 263), 1996, p. 62, nº 102.

19. Sur l'idée d'un sujet de droit «technique», voir R. Demogue, «La notion de sujet de droit», RTD civ., 1909, p. 611.

20. A. Bertrand-Mirkovic, La notion juridique de personne..., $\mathrm{n}^{\circ}$ 514, p. 263.

21. C. Labrusse-Riou, «L'embryon humain: qualifications juridiques et politique législative ", Revue générale de droit médical, $\mathrm{n}^{\circ}$ spécial, La recherche sur l'embryon: qualification et enjeux, p. 169.

22. N. Molfessis, «La dignité de la personne humaine en droit civil», in La Dignité de la personne humaine, p. 134, $\mathrm{n}^{\circ}$ 35. Comp. G. Loiseau, «Pour un droit des choses», Dalloz, 2006, p. 3015-3020. 


\section{A. L'absence de personnalité juridique du fœtus}

Le statut juridique de l'enfant simplement conçu soulève des interrogations ${ }^{23}$ tant juridiques, que morales, philosophiques et religieuses. Pour beaucoup, les qualités de personne juridique et de personne humaine seraient intrinsèquement liées. Refuser de qualifier le fotus de personne écarterait toute possibilité de protection. Ne paraîtil pas choquant de classer dans la catégorie des choses un être humain qui, s'il ne vit pas encore de manière autonome, n'en est pas moins déjà en vie dès sa conception? N'est-il pas autre chose qu'un simple amas de cellules?

Certains éléments semblent militer pour une personnification de l'enfant simplement conçu. Certes, le fotus n'a pas de patrimoine propre depuis que le curateur au ventre a été supprimé en $1964^{24}$. Toutefois, les articles 725 et 906 du Code civil prévoient qu'il peut être le bénéficiaire d'une donation ou d'un testament. L'article L. 132-8 du Code des assurances autorise la stipulation pour autrui au profit des «enfants nés ou à naître». L'adage infans conceptus, hérité du droit romain, permet, de manière plus générale, de réputer le foetus né dès qu'il y va de son intérêt. Cette règle a été érigée en principe général du droit par la Cour de cassation dans l'arrêt Euravie du 10 décembre $1985^{25}$. L'application de ce principe suppose que l'enfant naisse ensuite vivant et viable. Néanmoins, n'est-il pas possible de considérer que le fotus a d'ores et déjà une capacité à recevoir sous condition résolutoire $^{26}$ ? Apte à recevoir dès sa conception, il perdrait rétroactivement ce droit dans l'hypothèse où il ne naîtrait pas vivant et viable.

De nombreux auteurs sont favorables à une admission de la personnalité juridique de l'embryon ${ }^{27}$. Sans aller aussi loin, d'autres envisagent de créer une troisième catégorie ${ }^{28}$, entre les personnes et les choses. La traditionnelle summa divisio entre les personnes et les choses ne serait plus d'actualité face aux avancées de la science ${ }^{29}$.
L'embryon n'est-il pas déjà une «personne potentielle» ${ }^{30}$, une personne «en devenir ${ }^{31}$, ou encore une «personne par destination ${ }^{32}$ ?

La réglementation relative à l'établissement d'un acte d'enfant sans vie ne conduit-elle pas d'ailleurs à reconnaître l'individualité de l'enfant mort-né? Depuis la loi du 8 janvier 1993, l'article 79-1 du Code civil prévoit la possibilité pour les parents de déclarer à l'officier d'État civil des enfants nés sans avoir vécu. Un tel enregistrement officiel du décès a d'abord été réservé aux enfants ayant franchi un certain seuil de viabilité. Aux termes d'une circulaire du 30 novembre 2001, aucune déclaration n'était autorisée avant 22 semaines d'aménorrhée ou lorsque le fotus pesait moins de 500 grammes. Par trois arrêts rendus le 6 février 2008, la première chambre civile de la Cour de cassation a écarté cette règle en affirmant que l'établissement d'un acte d'enfant sans vie n'est pas conditionné par le niveau de développement du fotus ${ }^{33}$. Deux décrets et deux arrêtés du 20 août 2008 ont consacré cette solution, n'écartant l'établissement d'un tel acte qu'en cas de "fausse couche précoce» ou d' «interruption volontaire de grossesse». Une nouvelle circulaire interministérielle du 19 juin 2009 réintroduit un délai, de 15 semaines d'aménorrhée. Le caractère interprétatif de ce critère devrait le mettre à l'abri d'une nouvelle censure de la Cour de cassation ${ }^{34}$.

Grâce à l'établissement d'un acte d'enfant sans vie, l'enfant mort-né peut recevoir un prénom si ses parents le souhaitent. Son existence est officiellement constatée par une inscription sur les registres de l'État civil. L'enfant est par ailleurs inscrit dans une généalogie, l'acte comportant les noms et prénoms des père et mère et étant retranscrit dans un livret de famille. Des funérailles peuvent être organisées. N'est-ce pas par là même admettre que le foetus, même lorsqu'il ne naît pas vivant et viable, ne peut être assimilé à une chose?

Le fait que la loi organise une protection particulière de l'embryon n'implique-t-il pas, lui aussi, que la

23. J.-M. Roux, «Un sujet toujours en quête de son personnage: l'embryon humain", in Le droit de la biologie humaine. Vieux débats, nouveaux enjeux, A. Sériaux (dir.), Paris, Ellipses, 200o, p. 5 sq. A. Catherine, «L'assimilation de l'embryon à l'enfant? Les indices civilistes de personnification de l'embryon", Cahiers de la recherche sur les droits fondamentaux, n ${ }^{5}$, 2006, p. 79-96.

24. Ancien article 393 du Code civil de 1804: «Si, lors du décès du mari, la femme est enceinte, il sera nommé un curateur au ventre par le conseil de famille. À la naissance de l'enfant, la mère en deviendra tutrice, et le curateur en sera de plein droit le subrogé tuteur».

25. Cass. $1^{\text {re }}$ civ., 10 décembre 1985, Bull. civ., $\mathrm{n}^{\circ} 339$

26. En ce sens, D. Vigneau, "Dessine-moi” un embryon», Les Petites Affiches, 14 décembre 1994, p. 62-69.

27. N. Massager, Les Droits de l'enfant à naître, Bruxelles, Bruylant, 1997, p. 389, $\mathrm{n}^{\circ}$ 206. R. Andorno, La distinction juridique entre les personnes et les choses..., p. $289, \mathrm{n}^{\circ} 520$.

28. C. Sureau, «L'être prénatal. Illusion biologique, réalité humaine ou enjeu politique? », in Science, éthique et droit, N. M. Le Douarin et C. Puigelier (dir.), Paris, Odile Jacob, 2007, p. 217.

29. Marie-Angèle Hermitte parle ainsi d' «être à statut intermédiaire» concernant l'embryon («L'embryon humain, la science et le droit. Essai de chronologie", Revue générale de droit médical, $\mathrm{n}^{\circ}$ spécial, La recherche sur l'embryon: qualification et enjeux, p. 23). Voir aussi E. Sabathié, La Chose en droit civil, $\mathrm{n}^{\circ} 593$, p. 538 .

30. Comité consultatif national d'éthique, avis $\mathrm{n}^{\circ} 1$, Sur les prélèvements de tissus d'embryons et de foetus humains morts, à des fins thérapeutiques, diagnostiques et scientifiques, du 22 mai 1984. B. Baertschi, La valeur de la vie humaine et l'intégrité de la personne, Paris, PUF (Philosophie morale), 1995, p. 197: «L'être humain passe du statut de non-personne à celui de personne potentielle lors de la formation de son néocortex, et il ne devient une personne que plus tard, sans doute quelque temps après sa naissance».

31. CA Riom, 7 septembre 1995, cité par Cass. crim., 27 novembre 1996, Bull. crim., $\mathrm{n}^{\circ}$ 431, $2^{\mathrm{e}}$ arrêt.

32. X. Labbée, Condition juridique du corps humain. Avant la naissance et après la mort, Lille, Presses universitaires de Lille, 1990, p. 256: le fœetus serait une personne par destination du fait de son attache au corps de la mère.

33. Cass. $1^{\text {re }}$ civ., 6 février 2008, JCP, 2008, II 10045, note G. Loiseau.

34. G. Raoul-Cormeil, «D'une circulaire à l'autre pour réglementer l'acte d'enfant sans vie», L'essentiel du droit des personnes et de la famille, $\mathrm{n}^{\circ}$ 8, 2009 , p. 2. 
personnalité juridique lui soit attribuée? La loi du 17 janvier 1975 relative à l'avortement, si elle offre à la mère la possibilité d'avorter, encadre strictement cette pratique au-delà d'un certain délai. Son article premier, repris par l'article 16 du Code civil, affirme le respect dû à tout être humain. N'est-ce pas là reconnaître que le fotus est un être humain et, partant, une personne ${ }^{35}$ ? D'ailleurs, les textes relatifs à la procréation médicalement assistée envisagent l' «accueil» d'un embryon par un autre couple, et non son «don», terme réservé aux choses, tels les gamètes.

Si l'argumentation est séduisante, elle découle cependant d'une confusion entre les notions de personne juridique et de personne humaine. La personnalité juridique ne commence qu'avec la naissance. L'enfant simplement conçu n'est pas une personne juridique. Il s'agit nécessairement d'une chose ${ }^{36}$. Le droit positif ne laisse place à aucune catégorie intermédiaire. Il semble bien excessif de considérer que l'adage infans conceptus rattache le début de la personnalité juridique à la conception ${ }^{37}$. Les conséquences patrimoniales qui en résultent sont, en effet, soumises à la condition que l'enfant naisse vivant et viable. Pour que l'évènement rétroagisse, encore faut-il qu'il ait lieu. La naissance n'est pas, ici, une condition résolutoire mais une condition suspensive ${ }^{38}$.

Il peut certes paraître gênant de qualifier le fotus de chose sur un plan philosophique ou moral. La notion de chose est cependant ici purement juridique. Elle ne doit pas choquer, car elle ne conduit aucunement à nier sa qualité de personne humaine. L'embryon est, dès sa conception, une personne humaine à part entière. Il n'y a pas de sens à parler de personne potentielle. Il s'agit d'un être humain, mais sans personnalité juridique ${ }^{39}$. Une telle situation, si elle peut aujourd'hui surprendre, a pourtant pu exister par le passé. L'esclave, ou encore la personne frappée de mort civile ${ }^{40}$, étaient dépourvus de personnalité juridique. L'individu frappé de mort civil était réputé mort aux yeux de la loi : il perdait ses droits civils, politiques et civiques, sa succession était ouverte, et son mariage dissous ${ }^{41}$. Les esclaves étaient, quant à eux, pour le droit, des biens, objets de propriété, définis comme des meubles par le Code noir ${ }^{42}$. Il s'agissait pourtant d'êtres humains. La personnalité juridique est une abstraction détachée de la personne humaine.

Les concepts d'être et de chose ne sont pas incompatibles ${ }^{43}$. La notion de chose ne vise pas seulement les éléments inanimés. L'animal, qui est sans conteste un être vivant, est pourtant en droit positif une chose ${ }^{44}$. L'article 528 du Code civil ${ }^{45}$ le classe parmi les meubles par nature, tandis que l'article $524{ }^{46}$ prévoit même sa possible immobilisation par destination. L'animal n'est pas un sujet de droit. En tant qu' "être sensible » ${ }^{47}$, il bénéficie toutefois d'un certain nombre de dispositions protectrices ${ }^{48}$. L'existence de règles particulières ne saurait donc être un argument en faveur de la personnalité juridique du fœetus. Toutes les choses ne sont pas, en effet, traitées de la même manière. Le fœtus serait ainsi une chose spécialement protégée ${ }^{49}$, une chose sacrée ${ }^{50}$, du fait de sa nature d'être humain, de personne humaine ${ }^{51}$.

La jurisprudence récente en matière d'homicide involontaire du foetus est dès lors surprenante. Si un tel crime avait pu être admis dans le passé ${ }^{52}$, la chambre criminelle de la Cour de cassation a, dans un arrêt rendu le 30 juin

35. En ce sens, M.-A. Hermitte, «L'embryon humain, la science et le droit. Essai de chronologie», p. 18.

36. I. Moine, Les choses hors commerce..., p. 119. J.-C. Honlet, "Adaptation et résistance des catégories de droit privé», in Le droit saisi par la biologie, des juristes au laboratoire, C. Labrusse-Riou (dir.), Paris, LGDJ (Bibliothèque de droit privé, t. 259), 1996, p. 254. N.-J. Mazen et C. Taglione, "Les enjeux juridico-économiques de la recherche sur l'embryon humain», Revue générale de droit médical, $\mathrm{n}^{\circ}$ spécial, La recherche sur l'embryon: qualification et enjeux, p. 187.

37. C. Sureau, «L'être prénatal. Illusion biologique, réalité humaine ou enjeu politique? », p. 205.

38. J.-P. Gridel, Introduction au droit et au droit français, Paris, Dalloz, 1994, p. 727.

39. F. Bellivier et P. Egea, «L'être humain sans qualité», in Bioéthique, biodroit, biopolitique. Réflexions à l'occasion du vote de la loi du 4 août 2004 , S. Hennette-Vauchez (dir.), Paris, LGDJ (Droit et société), 2006, p. 121. A. Mirkovic, «Statut de l’embryon, la question interdite! », JCP, 2010, p. 99.

40. Pour un rapprochement avec la situation du fotus, voir A. Terrasson de Fougères, "La résurrection de la mort civile», RTD civ., 1997, p. 893.

41. Anciens articles 22 à 33 du Code civil, abrogés par la loi du 31 mai 1954.

42. Article $44 \mathrm{du}$ Code noir: «Déclarons les esclaves être meubles et comme tels entrer dans la communauté, n’avoir point de suite par hypothèque, se partager également entre les cohéritiers [...]».

43. Contra M.-A. Hermitte, «L'embryon humain, la science et le droit. Essai de chronologie», p. 24.

44. R. Libchaber, «Perspectives sur la situation juridique de l'animal», RTD civ., 2001, p. 239. Défavorable à une telle solution, voir J.-P. Marguénaud, "Droit des animaux: on en fait trop ou trop peu? », Dalloz, 2010, p. 816; J.-P. Marguénaud, L'Animal en droit privé, Paris, PUF, 1992.

45. Article $528 \mathrm{du}$ Code civil: «Sont meubles par leur nature les animaux et les corps qui peuvent se transporter d'un lieu à un autre, soit qu'ils se meuvent par eux-mêmes, soit qu'ils ne puissent changer de place que par l'effet d'une force étrangère».

46. Article 524 du Code civil: «Les animaux et les objets que le propriétaire d'un fonds y a placés pour le service et l'exploitation de ce fonds sont immeubles par destination [...]».

47. Selon les termes de l'article 9 de la loi du 10 juillet 1976 relative à la protection de la nature, JO du 13 juillet 1976, p. 4203 sq.

48. En droit interne, dès 1850, la loi Grammont punit, par exemple, le fait d'avoir exercé abusivement des mauvais traitements envers les animaux domestiques. Le décret du 7 septembre 1959 supprime la condition que les mauvais traitements aient été infligés en public, l'animal devenant l'objet même de la protection.

49. N.-J. Mazen et C. Taglione, «Les enjeux juridico-économiques de la recherche sur l'embryon humain», p. 190.

50. X. Labbée, «La valeur de l'embryon congelé», Dalloz, 2004, p. 1051.

51. C. Labrusse-Riou, «L'embryon humain: l'irreprésentable convoité», Écrits de bioéthique, Paris, PUF, 2007, p. 163: «Il ne s'agit pas de reconnaître des droits aux embryons mais d'abord de fonder des obligations envers eux en tant qu'ils sont partie intégrante de l'espèce humaine».

52. CA Douai, 2 juin 1987, JCP, 1989, II 21250, note X. Labbée. 
$1999^{53}$, affirmé que l'interprétation stricte de la loi pénale s'y oppose. Le fotus n'étant pas une personne, il ne pourrait être concerné par les atteintes à la personne sanctionnées par le droit pénal. Très contestée, la solution a été réaffirmée solennellement le 29 juin 2001 en assemblée plénière ${ }^{54}$. Au contraire, un homicide involontaire est reconnu lorsqu'un acte, commis au cours de la grossesse, a conduit à la mort de l'enfant lorsque ce dernier est né vivant et viable. Peu importe alors que sa vie extrautérine n'ait duré qu'un bref instant ${ }^{55}$. Sa naissance lui a permis d'accéder au rang de personne, susceptible, dès lors, d'être la victime d'un homicide. Pourtant, la personne ici protégée par le droit pénal n'est-elle pas la personne humaine, l'être de chair et de sang ${ }^{56}$ ?

Le fotus, s'il n'est pas une personne juridique, est un être humain, qui doit, en tant que tel, être respecté.

\section{B. Le fœtus, un être humain digne de respect}

L'enfant simplement conçu n'étant pas un sujet de droit, il ne jouit d'aucun droit subjectif. Personne humaine, le respect de sa dignité doit toutefois être assuré. Bien qu'elle légalise l'avortement, la loi du 17 janvier 1975 le présente comme une exception au principe selon lequel est garanti "le respect de tout être humain dès le commencement de sa vie». Elle ne remet aucunement en cause la qualité d'être humain de l'enfant dès sa conception. Le fotus de moins de 12 semaines est nécessairement concerné par le principe de respect dû aux êtres humains. Sinon pourquoi faudrait-il expressément prévoir une exception le concernant? Dans sa décision du 15 janvier 1975 relative à cette $\operatorname{loi}^{57}$, le Conseil constitutionnel reconnaît que l'embryon fait partie des êtres humains devant être respectés dès le commencement de leur vie ${ }^{58}$. Le Conseil admet cependant une possible dérogation à ce principe en cas de grande détresse de la mère.
Un embryon est un être humain digne de respect. Tant le droit français ${ }^{59}$ que le droit européen ${ }^{60}$ interdisent ainsi de créer des embryons pour la recherche scientifique. Une telle production, purement utilitaire, conduirait à nier leur qualité de personne humaine en les réduisant au rang de matériel biologique indifférencié et fongible n'ayant nullement vocation à donner naissance à un enfant. Un embryon ne peut non plus être conçu ou utilisé à des fins commerciales ou industrielles. Il ne s'agit pas d'une marchandise. Sa commercialisation est interdite ${ }^{61}$.

En tant qu'être humain, l'embryon ne peut être librement détruit. La loi du 17 janvier 1975 encadre strictement sa destruction, qui ne peut avoir lieu, au-delà de 12 semaines, qu'en cas de nécessité thérapeutique. De telles dispositions ne concernent toutefois que l'embryon in utero. Les règles relatives à la procréation médicalement assistée conduisent à une contestable réification de l'embryon in vitro ${ }^{62}$. Comment admettre qu'un être humain soit automatiquement détruit au bout de 5 années? Le fait de produire des embryons surnuméraires probablement voués à la destruction n'est-il pas contraire à la dignité humaine? Les embryons créés et congelés ne sont-ils pas de simples objets soumis au pouvoir de leurs «parents " ${ }^{63}$, lesquels peuvent, selon leur bon vouloir, décider de leur implantation, de leur don à un autre couple, de leur don à la recherche ou même de leur destruction? Un tel système «conduit à ce que l'attribution de la qualité d'être humain ne résulte pas d'une qualité ontologique, mais de la reconnaissance par un tiers (en l'espèce les parents biologiques). La possibilité pour un être humain d'en disqualifier un autre [...] entre en contradiction avec le principe de dignité $»{ }^{64}$.

Bien qu'encadrée, la recherche sur l'embryon semble, elle aussi, incompatible avec l'idée que l'embryon est une personne humaine ${ }^{65}$. L'embryon in vitro peut parfaitement faire l'objet de recherches ne présentant pour lui aucun intérêt personnel. À l'issue des recherches, l'embryon,

53. Cass. crim., 30 juin 1999, Dalloz, 1999, p. 710, note D. Vigneau; Dalloz, 200o, p. 181, note G. Roujou de Boubé et B. de Lamy; p. 27, note Y. Mayaud; p. 169, note C. Desnoyer et L. Dumaire; Droit pénal, 200o, p. 4, note M.-L. Rassat; Les Petites Affiches, 17 novembre 1999, note F. Debove; Revue de droit sanitaire et social, 2000, p. 88, note G. Mémeteau et M. Harichaux.

54. Cass. assemblée plénière, 29 juin 2001, Dalloz, 2001, p. 2917, note Y. Mayaud; p. 2907, note J. Pradel; RTD civ., 2001, p. 560, note J. Hauser; Revue de science criminelle, 2002, p. 97, note B. Bouloc; Revue de droit sanitaire et social, 2001, p. 829, note A. Terrasson de Fougères. Solution constante depuis: Cass. crim., 25 juin 2002, Bull. crim., $\mathrm{n}^{\circ} 144$.

55. Cass. crim., 2 décembre 2003, Bull. crim., $\mathrm{n}^{\circ}$ 230. Cass. crim., 23 octobre 2001, Bull. crim., $\mathrm{n}^{\circ} 217$.

56. C. Levy, «De la distinction entre "personne” et "être humain” en droit pénal: l'enfant à naître n'est pas "autrui” au sens du droit pénal», Revue générale de droit médical, $\mathrm{n}^{\circ}$ spécial, La recherche sur l'embryon: qualification et enjeux, p. 48; J. Sainte-Rose, «La protection pénale de l'enfant à naître», in Interrogations sur l'évolution des droits fondamentaux de la personne humaine en 2001 et 2002, G. Lebreton (dir.), Paris, L'Harmattan, 2004, p. 7-20.

57. Déc. $\mathrm{n}^{\circ}$ 74-54 DC du 15 janvier 1975, Loi relative à l'interruption volontaire de la grossesse, JO du 16 janvier 1975, p. 671. Voir aussi déc. $\mathrm{n}^{\circ}$ 2001-446 DC du 27 juin 2001, Loi relative à l'interruption volontaire de grossesse et à la contraception, JO du 7 juillet 2001, p. 10828, où le Conseil constitutionnel examine la compatibilité de l'allongement du délai légal dans lequel une IVG peut être pratiquée avec la dignité humaine.

58. B. Mathieu, «Les droits fondamentaux... », p. 222

59. Article L. 2151-2 du CSP.

60. Convention européenne sur les Droits de l'homme et la biomédecine, signée à Oviedo le 4 avril 1997, article 18-2 ${ }^{\circ}$.

61. Article L. 2162-1 du Code de la santé publique.

62. C. Neirinck, «L'embryon humain ou la question en apparence sans réponse de la bioéthique », Les Petites Affiches, 9 mars 1998, p. 4.

63. M.-A. Hermitte, «L'embryon humain, la science et le droit. Essai de chronologie», p. 34. C. Neirinck, «L'embryon humain: une catégorie juridique à dimensions variables? ", Dalloz, 2003, p. 841 sq., spécialement la conclusion. G. Mémeteau, «L'embryon législatif», Dalloz, 1994, p. 355.

64. B. Mathieu, «Les droits fondamentaux... », p. 224

65. B. Mathieu, «La recherche sur l'embryon au regard des droits fondamentaux constitutionnels», Dalloz, 1999, p. 451. 
vulgaire matériau de laboratoire, ne pourra plus être implanté. Sa destruction est obligatoire ${ }^{66}$. La dignité de l'être humain n'est-elle pas niée dès lors que l'embryon, objet d'expérimentation, est inexorablement voué à être détruit? L'admission du diagnostic préimplantatoire pour autrui ${ }^{67}$ réduit, quant à elle, l'embryon au rang de «bébémédicament». N'est-il pas conçu comme un simple instrument de guérison d'un autre être humain, comme le moyen de satisfaire les intérêts d'autrui? Il semble difficile de parler d'être humain face à une telle logique utilitariste ${ }^{68}$. L'enfant n'est pas voulu pour lui-même, mais seulement dans le but de soigner son aîné. La qualité d'être humain semble incompatible avec de telles pratiques. Le Conseil constitutionnel a d'ailleurs affirmé, dans sa décision du 27 juillet $1994^{69}$, que l'embryon in vitro n'est pas un être humain. Il y indique que «le législateur [...] n'a pas considéré que devait être assurée la conservation, en toutes circonstances et pour une durée indéterminée, de tous les embryons déjà formés; qu'il a estimé que le principe du respect de tout être humain dès le commencement de sa vie ne leur était pas applicable; qu'il a, par suite, nécessairement considéré que le principe d'égalité n'était pas non plus applicable à ces embryons".

Une telle distinction entre embryons in vitro et in utero surprend. Elle ne figure nullement dans la loi. On se demande d'ailleurs en quoi le mode de procréation peut influer sur la qualité d'être humain de l'enfant conçu ${ }^{70}$.

Tout embryon est, dès sa conception, un être humain. Tout comme l'enfant simplement conçu, le cadavre est, juridiquement, une chose. Il s'agit cependant, là encore, d'un être humain digne de respect.

\section{Le respect dû à l'être humain après la mort}

«Les morts ne sont plus des personnes, ils ne sont plus rien ». Cette célèbre citation de Marcel Planiol est exces$\operatorname{sive}^{71}$. La mort marque certes la fin de la personnalité juridique. La personne humaine décédée est une chose (A). Son appartenance à l'espèce humaine ne disparaît pas pour autant et justifie que des égards particuliers lui soient rendus (B).

\section{A. Le cadavre, une chose sacrée}

Après la mort, la personnalité juridique s'éteint. Le corps du défunt est, lui, toujours présent. N'étant plus le support d'une personne juridique, le cadavre est désormais une chose. Certains ont voulu défendre l'idée d'une demipersonnalité des personnes décédées ${ }^{72}$. Tant leur dépouille que leur mémoire sont en effet protégées par le droit. La jurisprudence n'a-t-elle pas parfois semblé admettre une atteinte à leur vie privée ${ }^{73}$ ? Dans l'affaire Gabin, la saisie du journal reproduisant la photographie du défunt sur son lit de mort fut ordonnée par le juge des référés aux motifs que «le droit au respect de la vie privée s'étend par-delà la mort à celui de la dépouille mortelle; que cette atteinte constitue une immixtion considérable dans l'intimité du disparu ${ }^{74}$.

Parler de demi-personnalité n'a pourtant pas de sens. On ne peut considérer qu'une part de la personnalité subsiste après la mort, le défunt ayant droit au respect de son corps. Seuls les hommes vivants sont des sujets de droit. Ce n'est pas parce que des règles particulières de protection sont prévues, qu'une personne juridique est automatiquement reconnue par le droit. Un objet de droit peut parfaitement être soumis à un statut particulier, de même que les animaux par exemple ${ }^{75}$. Le corps d'un mort est une chose ${ }^{76}$. Des têtes maories ont ainsi pu être considérées comme pouvant faire l'objet d'un régime particulier de domanialité publique ${ }^{77}$, ou un cadavre l'«objet d'un droit de copropriété familial ${ }^{78}$. La profanation du corps d'une jeune fille récemment inhumée est, pour le droit pénal, une «violation de sépulture» et non un viol ou un attentat à la pudeur, ce qui démontre bien là encore l'assimilation du cadavre à une chose.

Les morts relèvent désormais de la catégorie des choses et non des personnes. Ils ne peuvent être titulaires de droits de la personnalité, réservés aux seules personnes juridiques. Il est impossible de parler d'atteinte à leur vie privée ou à leur droit à l'image. Seule la famille du défunt peut invoquer son droit à l'intimité de la vie

66. Article L. 2151-5 du CSP.

67. Article L. 2131-4-1 du CSP.

68. J. Clercks, «L'embryon humain. Le législateur, le début de la vie et la loi relative à la bioéthique », Revue du droit public, $\mathrm{n}^{\circ} 3,2006, \mathrm{p} .737$.

69. Déc. ${ }^{\circ} 94-343 / 344$ DC du 27 juillet 1994, JO du 29 juillet 1994, p. 11024.

70. C. Labrusse-Riou, «L'embryon humain: qualifications juridiques et politique législative», p. 168. B. Mathieu, «La dignité de la personne humaine: quel droit? quel titulaire? ", Dalloz, 1996, p. 282.

71. M. Planiol, Traité élémentaire de droit civil, $12^{\mathrm{e}}$ édition, Paris, LGDJ, 1935, t. I, p. 152, \$371. Elle fut d'ailleurs supprimée par la suite.

72. R. Demogue, «La notion de sujet de droit», RTD civ., 1909, p. 611. G. Timbal, La Condition juridique des morts, Thèse, Université de Toulouse, 1902.

73. Cass. crim., 20 octobre 1998, Dalloz, 1999, p. 106, note B. Beignier. Cass. crim., 21 octobre 1980, Bull. crim., nº 262.

74. JCP, 1977, 18711, note D. Ferrier.

75. Voir supra.

76. J. Carbonnier, «Sur les traces du non-sujet de droits», Flexible droit. Pour une sociologie du droit sans rigueur, 10 édition, Paris, LGDJ, 2001, p. 233. X. Labbée, La Condition juridique du corps humain avant la naissance et après la mort, Lille, Presses universitaires de Lille, $1990, \mathrm{p} .167$ sq. M. Wagner, "Quelques réflexions autour de l'article 16-1-1 du Code civil: vers une obligation générale de respect du corps humain», Revue de la recherche juridique, $\mathrm{n}^{\circ} 4,2009$, p. 1874 .

77. TA Rouen, 27 décembre 2007 , JCP, 2008, II 10041, note C. Saujot.

78. TGI Lille, 5 décembre 1996, Dalloz, 1997, p. 376, note X. Labbée. 
privée, sur le fondement de l'article 9 du Code civil. Selon la Cour de cassation, «le droit d'agir pour le respect de la vie privée s'éteint au décès de la personne concernée, seule titulaire de ce droit ${ }^{79}$.

Le cadavre est toutefois une chose particulière, une chose sacrée ${ }^{80}$, insusceptible d'être traitée comme une chose ordinaire. Le caractère sacré du cadavre n'a aucune connotation religieuse. Comme le précise Samuel Pufendorf dans son traité de droit naturel, «il n'y a point de qualité morale ou de sainteté qui y soit attachée. Tout ce qu'emporte pareil épithète, c'est que les hommes ne sont tenus de se servir de cette chose que d'une certaine manière ${ }^{81}$. Après la mort, le corps est une chose sacrée, au sens antique du terme, c'est-à-dire une chose séparée du reste car spécialement protégée.

\section{B. Le défunt, un être humain digne de respect}

S’il est une chose, le cadavre est néanmoins digne de respect. La mémoire des défunts peut être protégée, sur le fondement du respect dû à la dignité humaine ${ }^{82}$. Conçu comme visant à protéger l'espèce humaine, ce principe semble applicable à tous les êtres humains, même décédés ${ }^{83}$. La dignité des morts est une limite posée à la liberté d'expression des journalistes ${ }^{84}$. Dans l'affaire Érignac ${ }^{85}$, la Cour de cassation a clairement indiqué que la publication d'une photographie représentant «distinctement le corps et le visage d'(un) préfet assassiné » était «attentatoire à la dignité de la personne humaine». Un arrêt rendu le $1^{\text {er }}$ juillet 2010 par la première chambre civile de la Cour de cassation ${ }^{86}$ indique, lui aussi, que la publication de la photographie d'un otage mort sous la torture est «contraire à la dignité humaine» et constitue «une atteinte à la mémoire ou au respect dû au mort».

Le corps des personnes décédées est, lui aussi, protégé. Le respect de la dépouille mortelle se voit assuré par le droit pénal. L'article 225-17 du Code pénal incrimine toute atteinte à l'intégrité physique du cadavre. La place de ce texte, au cour de dispositions relatives à la dignité de la personne, met en exergue la qualité de personne humaine du défunt, dont la dignité doit être respectée ${ }^{87}$. La protection pénale du cadavre s'inscrit dans celle, plus large, de la dignité de la personne humaine.

Sur le plan civil, l'article 16-1-1 du Code civil, issu de la loi du 19 décembre 2008, affirme que le respect dû au corps humain ne cesse pas avec la mort. Ce respect est un principe général, indépendant du concept de personnalité juridique. Auparavant, et en l'absence même de texte, la jurisprudence avait eu l'occasion d'appliquer une solution identique. Le Conseil d'État, dans l'arrêt Milhaud ${ }^{88}$ du 2 juillet 1993, avait ainsi indiqué que «les principes déontologiques fondamentaux relatifs au respect de la personne humaine, qui s'imposent au médecin dans ses rapports avec son patient, ne cessent pas de s'appliquer avec la mort de celui-ci».

L'intégrité physique du cadavre doit être préservée. La réalisation d'identification génétique post mortem est aujourd'hui interdite en droit français. La question avait fait l'objet de vifs débats à la suite de l'affaire Montand ${ }^{89}$. Lors de la révision des lois relatives à la bioéthique en 2004, un nouvel article 16-11 alinéa 2 du Code civil précisa qu'une telle mesure n'était envisageable qu'en cas de consentement préalable de la personne concernée de son vivant. La position de la CEDH n'est pas aussi stricte. Par un arrêt Jäggi c. Suisse du 13 juillet $2006^{90}$, celle-ci a estimé que le refus d'autoriser, à la demande de l'enfant probable, l'exhumation du cadavre d'un père éventuel pour procéder au prélèvement d'ADN avait constitué une violation de l'article 8 de la $\mathrm{CEDH}$ en portant atteinte au droit à l'identité, dont relève le droit à connaître son ascendance, lequel fait partie intégrante de la vie privée. Dans un arrêt du 5 mai 2009, la Cour n'a toutefois pas étendu ce droit à l'exhumation du corps des grands-parents potentiels ${ }^{91}$.

La récente exposition Our body, à corps ouvert a été l'occasion de réaffirmer le respect dû au cadavre et le principe de sa non-commercialité. Cette exposition consistait à présenter au public de véritables corps humains dans des postures diverses par le procédé de plastination. Dans un arrêt en date du 16 septembre $2010^{92}$, la première chambre civile de la Cour de cassation a rappelé qu' «aux termes de

79. Cass. $1^{\text {re }}$ civ., 14 décembre 1999, Dalloz, 2000, p. 372, note B. Beignier. Cass. $2^{\mathrm{e}}$ civ., 20 novembre 2003, Bull. civ., II n ${ }^{\circ} 354$. Cass. $2^{\mathrm{e}}$ civ., 8 juillet 2004, Dalloz, 2004, p. 2088.

80. X. Labbée, note sous TGI Lille, 5 décembre 1996, Dalloz, 1997, p. 376. E. Sabathié, La Chose en droit civil, nº 603, p. 548. M. Wagner, «Quelques réflexions autour de l'article 16-1-1... », p. 1874 ; H. Popu, "Le corps humain post mortem, une chose "extra-ordinaire" ", Revue de la recherche juridique, 2009-1, p. 229 sq.

81. S. von Pufendorf, Droit de la nature et des gens, Lyon, 1771, p. 16 [réédition: S. von Pufendorf, Le Droit de la nature et des gens, Jean Barbeyrac (trad.), Caen, Presses universitaires de Caen, 2010].

82. G. Mémeteau, "Vie biologique et personnalité juridique. “Qui se souvient des hommes?” ", in La Personne humaine, sujet de droit, 4 e journées René Savatier (Poitiers, 25 et 26 mars 1993), Paris, PUF, 1994, p. 32.

83. Contra P.-J. Delage, note sous Cass. $1^{\text {re }}$ civ., $1^{\text {er }}$ juillet 2010, Dalloz, 2010, p. 2047.

84. Cass. $2^{\mathrm{e}}$ civ., 4 novembre 2004, Dalloz, 2005, p. 696, note J. Corpart.

85. Cass. $1^{\text {re }}$ civ., 20 décembre 2000, Dalloz, 2001, p. 872, note J.-P. Gridel.

86. Cass. $1^{\text {re }}$ civ., $1^{\text {er }}$ juillet 2010, Dalloz, 2010, p. 2044, note P.-J. Delage; JCP, 2010, 942, note G. Loiseau.

87. C. Labrusse-Riou, «L'humain en droit: réalité, fiction, utopie? », Écrits de bioéthique, p. 386-416. C. Labrusse-Riou, «Droit des personnes et de la famille", in Libertés et Droits fondamentaux, $2^{\mathrm{e}}$ édition, M. Delmas-Marty et C. Lucas de Leyssac (dir.), Paris, Seuil, 2002, p. 333.

88. CE, 2 juillet 1993, JCP, 1993, II 22133, note P. Gonod.

89. CA Paris, 6 novembre 1997, Dalloz, 1998, p. 161, obs. H. Gaumont-Prat; RTD civ., 1998, p. 87, note J. Hauser.

90. CEDH, Jäggi c. Suisse, 13 juillet 2006, RTD civ., 2006, p. 727, obs. J.-P. Marguénaud.

91. CEDH, Menéndez Garcia c. Espagne, 5 mai 2009, RTD civ., nº 4, 2009, p. 679, \$ 2, obs. J.-P. Marguénaud et P. Remy-Corlay.

92. Cass. $1^{\text {re }}$ civ., 16 septembre 2010, JCP, 2010, $\mathrm{n}^{\circ}$ 1239, note B. Marrion; Dalloz, 2010, p. 2750, note G. Loiseau et p. 2754, note B. Edelman. 
l'article 16-1-1, alinéa 2, du Code civil, les restes des personnes décédées doivent être traités avec respect, dignité et décence», et "que l'exposition de cadavres à des fins commerciales méconnaît cette exigence». Elle approuve donc la Cour d'appel d'avoir interdit la poursuite de l'exposition, par application de l'article 16-2 du Code civil, permettant au juge de prendre toute mesure propre à faire cesser le trouble. Comme le tribunal de grande instance de Paris, la Cour de cassation fonde sa solution sur l'interdiction de commercialiser le cadavre. Les premiers juges avaient en effet, eux aussi, estimé que «la commercialisation des corps par leur exposition porte une atteinte manifeste au respect qui leur est dû ${ }^{93}$. La solution est identique à celle préconisée par le Comité consultatif national d'éthique qui, dans un avis du 7 janvier 2010, a considéré que les expositions de cadavres à des fins soi-disant anatomiques et pédagogiques sont « une forme d'exploitation du corps des morts à visée commerciale qui contrevient à l'esprit de la loi française ${ }^{94}$.

La Cour d'appel avait, elle aussi, interdit l'exposition en cause. Elle avait toutefois adopté une motivation totalement différente. Elle s'était seulement attachée à l'absence de consentement des personnes dont le cadavre était exposé. Chacun peut, en effet, librement prendre certaines décisions quant au devenir de son corps après sa mort. Il est loisible de choisir entre la crémation et l'inhumation, de refuser de donner ses organes post mortem, ou d'accepter de donner son corps à la science. Tout n'est cependant pas permis. La loi du 19 décembre 2008 a, par exemple, limité les lieux de conservation des cendres du cadavre $^{95}$. Il est notamment impossible de les garder dans un lieu privé ou de les disperser sur les voies publiques. Le Conseil d'État a, par ailleurs, refusé la possibilité de recourir à la cryogénisation ${ }^{96}$. Le consentement de la personne est donc parfois insuffisant. La Cour de cassation, concernant l'exposition Our body, à corps ouvert, ne reprend d'ailleurs pas les arguments avancés par la Cour d'appel et opère une substitution de motifs afin d'affir- mer que la finalité commerciale de la manifestation méconnaissait ici la dignité des morts. La dignité est conçue comme s'imposant à l'homme. Elle est susceptible de limiter sa liberté de choix. L'être humain ne peut porter atteinte à sa propre dignité, laquelle «implique pour l'individu l'obligation morale absolue de se plier au respect de soi ${ }^{97}$. La dignité est alors opposée à la liberté individuelle.

Deux conceptions de la dignité sont en effet concevables. La première, individualiste, est centrée sur l'individu. Elle fonde la dignité sur la liberté de l'homme. La dignité est alors définie comme «la valeur éminente qui s'attache à l'individu compte tenu du caractère unique, irremplaçable de son accomplissement personnel ${ }^{98}$. «Principe matriciel» ${ }^{99}$, la dignité serait la source de tous les autres droits fondamentaux. La seconde conception, dite spécifique, voit au contraire dans la dignité le moyen de préserver l'espèce humaine ${ }^{100}$, et non l'individu à titre personnel. Selon le préambule de la Charte des droits fondamentaux de l'Union européenne, «la jouissance de ces droits entraîne des responsabilités et des devoirs tant à l'égard d'autrui qu'à l'égard de la communauté humaine et des générations futures». Il est traditionnel d'admettre que la liberté de chacun est limitée par celle des autres. On semble cependant désormais s'orienter vers une analyse de la dignité humaine permettant de limiter la liberté de l'individu envers lui-même, dès lors que l'on considère que ses actes portent atteinte à l'humanité qui l'habite $^{101}$. Chaque être humain se verrait ainsi contraint de respecter la part d'humanité qui est en lui. La dignité est alors conçue comme un devoir et une charge, un respect que l'on se doit à soi-même ${ }^{102}$. La solution vaut également du vivant de la personne, comme le révèle la célèbre affaire du «lancer de nains » ${ }^{103}$. Le spectacle fut, en effet, interdit en dépit des arguments du nain, invoquant sa volonté de continuer à travailler de la sorte. N'est-ce pas aller trop loin? «De libératrice, (la dignité) devient liberticide ${ }^{104}$.

93. TGI Paris, 21 avril 2009, Dalloz, 2009, p. 1192, note X. Labbée.

94. Comité consultatif national d'éthique, Avis $\mathrm{n}^{\circ}$ 111, sur les problèmes éthiques posés par l'utilisation des cadavres à des fins de conservation ou d'exposition muséale, spécialement p. 14

95. Article L. 2223-18-2 du Code général des collectivités territoriales.

96. CE, 6 janvier 2006, Martinot, JCP, 2006, II 10059, note L. Erstein.

97. O. Cayla, «Jeux de nains, jeux de vilains», in Les droits fondamentaux de la personne humaine en 1995 et 1996, G. Lebreton (dir.), Paris, L'Harmattan, 1998, p. 155.

98. G. Lebreton, «Les ambiguïtés du droit français à l'égard de la dignité de la personne humaine», in Mélanges Patrice Gélard. Droit constitutionnel, P. Fraisseix et al. (dir.), Paris, Montchrestien, 2000, p. 55

99. B. Mathieu, «La dignité de la personne humaine: quel droit? quel titulaire? », p. 282.

100. En ce sens, B. Edelman, «La dignité de la personne humaine, un concept nouveau », Dalloz, 1997, p. 185. M. Fabre-Magnan, «Dignité humaine», in Dictionnaire des Droits de l'homme, H. Gaudin et al. (dir.), Paris, PUF (Quadrige), 2008, p. 285-291.

101. En ce sens, très critique, O. Cayla, "La négation de toute possible identité européenne par la Charte européenne des droits fondamentaux", in Regards critiques sur l'évolution des droits fondamentaux de la personne humaine en 1999 et 200o, G. Lebreton (dir.), Paris, L'Harmattan, 2002, p. 103-114.

102. N. Molfessis, «La dignité de la personne humaine en droit civil», p. 107 sq., spécialement $\mathrm{n}^{\circ} 28$.

103. CE, 27 octobre 1995, Commune de Morsang-sur-Orge, JCP, 1996, II 22630, note F. Hamon.

104. G. Lebreton, «Les ambiguïtés du droit français à l'égard de la dignité de la personne humaine», p. 55. Dans le même sens, E. Dreyer, «La dignité opposée à la personne», Dalloz, 2008, p. 2730; E. Dreyer, «Dignité (Droits fondamentaux et) ", in Dictionnaire des droits fondamentaux, D. Chagnollaud et G. Drago (dir.), Paris, Dalloz, 2010, p. 251; D. Roman, « “À corps défendant”. La protection de l'individu contre lui-même», Dalloz, 2007, p. 1284. 
En conclusion, avant la naissance comme après la mort, il est impossible de parler de personne au sens juridique du terme. Concernant les personnes physiques, la personnalité juridique n'est applicable qu'aux individus vivants nés viables. L'embryon et le cadavre relèvent nécessairement de la catégorie des choses. Retenir une telle qualification ne revient cependant pas à nier leur qualité de personne humaine. Il s'agit dès lors de choses dignes de respect. 